\title{
Susceptibilidad in vitro de Candida albicans y no albicans Aisladas de Prótesis Dentales de Pacientes con Estomatitis Protésica a Tres Sustancias de Desinfección
}

\author{
In vitro Susceptibility of Candida albicans and Non-albicans Isolated from \\ Dental Prothesis in Patients with Denture Stomatitis to Three Disinfectants
}

\author{
Diego Michel Castillo Sauceda*; Ma. Concepción Tello Zavala*; Luis Octavio Sánchez Vargas*; \\ Ma. Bertha Gómez Gutiérrez"; Nadya Nava-Zárate* \& Saray Aranda-Romo*
}

CASTILLO, S. D. M.; TELLO, Z. M. C.; SÁNCHEZ, V. L. O.; GÓMEZ, G. M. B.; NAVA-ZÁRATE, N. \& ARANDA-ROMO, S. Susceptibilidad in vitro de Candida albicans y no albicans aisladas de prótesis dentales de pacientes con estomatitis protésica a tres sustancias de desinfección. Int. J. Odontostomat., 9(3):373-377, 2015.

RESUMEN: A pesar del gran número de productos disponibles para la limpieza de prótesis dentales, el $60 \%$ de los portadores hace uso de ellos, posiblemente por la situación económica. El objetivo fue determinar la susceptibilidad antifúngica in vitro que presenta Candida y sus especies a tres sustancias utilizadas para la desinfección de prótesis (hipoclorito de sodio, ácido acético y solución de superoxidación). Se obtuvieron aislados clínicos de Candida de pacientes portadores de prótesis diagnosticados con estomatitis protésica, para su posterior estudio de susceptibilidad in vitro a las diferentes sustancias. El hipoclorito de sodio al 0,5\% mostró in vitro una mayor inhibición para las cepas de Candida albicans y Candida no albicans. El ácido acético y la solución de superoxidación no mostraron inhibición in vitro frente a ambas cepas. El hipoclorito de sodio al 0,5\% tiene un efecto inhibitorio in vitro sobre las cepas de C. albicans y Candida no albicans.

PALABRAS CLAVE: Candida albicans, estomatitis protésica, hipoclorito de sodio.

\section{INTRODUCCIÓN}

Cuando se introduce dentro de la cavidad oral una prótesis dental, Candida es capaz de adherirse y colonizarla, aumentando su frecuencia a un $66,7 \%$ en éstos pacientes (Williams et al., 2011). De acuerdo con varios estudios in vitro, la contaminación microbiana de la resina acrílica se produce rápidamente, y las levaduras se adhieren muy bien a la base de la prótesis (Jackson et al., 2014; Koch et al., 2013). Una vez que la Candida se adhiere sobre las superficies de éstas y coloniza, es capaz de desarrollar la formación de una comunidad de microorganismos unidos a una superficie y rodeados por una matriz extracelular polisacárida denominada biofilm (Williams et al.; Koch et al.). La transición de Candida, desde un comensal inocuo a organismo patogénico es compleja, y depende tanto de factores locales como sistémicos, que contribuyen al desarrollo de estomatitis protésica (EP), re- portándose $C$. albicans como el principal agente etiológico en el $71,4 \%$ de los casos. La EP también se asocia a la mala higiene de la prótesis, lo que demuestra la importancia de su limpieza empleando métodos mecánicos o químicos (Williams \& Lewis, 2000). Existen en el mercado diversos agentes utilizados para la desinfección de las prótesis de bajo costo, como el ácido acético $(A C)$, un líquido astringente que tiene potencial antimicrobiano. Estudios in vitro han demostrado que las bajas dosis fungicidas del ácido acético inducen la apoptosis de $C$. albicans. La inmersión total de dentaduras en una solución de AC al 10\% o $5 \%$ durante la noche, reduce las colonias (cfu/ml) de Candida en la saliva, así como la presencia de EP; el efecto de remoción es comparable con una solución de hipoclorito de sodio $(\mathrm{NaOCl})$ al $1 \%$ (Pinto et al., 2008).

\footnotetext{
* Laboratorio de Bioquímica, Microbiología y Patología, Facultad de Estomatología, UniversidadAutónoma de San Luis Potosí, San Luis Potosí, México. ** Laboratorio de Microbiología Facultad de Medicina ,Universidad Autónoma de San Luis Potosí, San Luis Potosí, México.
} 
CASTILLO, S. D. M.; TELLO, Z. M. C.; SÁNCHEZ, V. L. O.; GÓMEZ, G. M. B.; NAVA-ZÁRATE, N. \& ARANDA-ROMO, S. Susceptibilidad in vitro de Candida albicans y no albicans aisladas de prótesis dentales de pacientes con estomatitis protésica a tres sustancias de desinfección. Int. J. Odontostomat., 9(3):373-377, 2015.

El NaOCl, actúa como agente anti-fúngico cuando es empleado en solución para sumergir las prótesis en caso de EP, debido a que reduce la capacidad de adhesión de Candida a las células epiteliales (Estrela et al., 2013). Ha sido empleado por mucho tiempo como desinfectante protésico, ya que reduce el crecimiento microbiano sobre las superficies de la prótesis. Según estudios (Estrela et al.; Davi et al., 2010), la inmersión de la prótesis en $0,5 \%$ de $\mathrm{NaOCl}$, durante 5 minutos, es suficiente para eliminar $C$. albicans. Chau et al. (1995) establecen que una inmersión de 10 min en $\mathrm{NaOCl}$ al 5,25\% es efectiva para desinfectar superficies internas y externas del acrílico de la prótesis, sin embargo, debe emplearse una vez a la semana ya que decolora el acrílico y afecta su fuerza de flexión.

También existen agentes desinfectantes de costo elevado por ejemplo la solución electrolizada de súper-oxidación (SSO), una solución con $\mathrm{pH}$ neutro al 0,004\% (40 ppm) de Cl activo, con un amplio espectro antimicrobiano, esto se atribuye al efecto de oxidación de los grupos sulfhidrilos y aminoácidos de la pared bacteriana, lo que afecta el proceso de respiración y nutrición de los microorganismos, se ha demostrado su eficacia para inactivar $C$. albicans en estudios realizados in vitro en los cuales un tiempo de exposición de $30 \mathrm{~s}$ fue suficiente para inactivar completamente todas las muestras (Zeng et al., 2011; lieri et al., 2006).

El objetivo fue evaluar la susceptibilidad antifúngica in vitro sobre aislamientos clínicos de Candida obtenidos de las superficies de las prótesis dentales a 3 sustancias utilizadas para su desinfección.

\section{MATERIAL Y MÉTODO}

Ensayo de susceptibilidad in vitro. Se evaluaron soluciones desinfectantes de AC, $\mathrm{NaOCl}$ y SSO. Se utilizaron tres aislamientos de $C$. albicans y tres aislamientos de Candida no albicans. obtenidos previamente de las prótesis. Para determinar si el aislamiento correspondía a $C$. albicans se realizó la prueba del tubo germinal. Para la tipificación de Candida no albicans, se utilizaron placas de CHROMagar ${ }^{\circledR}$ siguiendo las especificaciones del fabricante, como control se emplearon cepas ATCC 24433 y ATCC 90028 de $C$. albicans. Se preparó una suspensión de cada una de las cepas de $C$. albicans y Candida no albicans a una concentración de 0,5 de McFarland utilizando un nefelómetro. Se hicieron diluciones de $\mathrm{NaOCl}$ $(5,25 \%)$ y $A C(5 \%)$ como sigue: $0,5,0,25,0,125$ y $0,062 \%$ en agua destilada estéril. La SSO se aplicó directamente sobre la placa de agar mediante una pipeta de volumen fijo ( $20 \mathrm{~mL}$ de SSO), debido a las instrucciones del fabricante. Con las diluciones preparadas se impregnaron discos de $6 \mathrm{~mm}$ de diámetro de papel filtro (Whatman ${ }^{\circledR} 40$, Whatman Ltd. England UK) durante 5 min con cada una de las sustancias a evaluar. Cada una de las cepas y aislamientos de $C$. albicans y Candida no albicans a evaluar se sembraron en placas de agar dextrosa sabouraud. En cada placa ya inoculada se colocaron los discos de papel impregnados, distribuidos en la parte central de cada placa, como control negativo se colocaron discos impregnados en agua estéril. Las placas se incubaron a $37^{\circ} \mathrm{C}$ y luego de $48 \mathrm{~h}$ de incubación se midió en milímetros el diámetro total de los halos de inhibición formados alrededor de cada disco.

Se realizó análisis descriptivo de las variables registradas utilizando medidas de tendencia central para las variables continuas y proporciones para las variables nominales.

\section{RESULTADOS}

Pruebas de sensibilidad in vitro de $C$. albicans y Candida no albicans a los diferentes desinfectantes.

El AC y la SSO a las diferentes concentraciones utilizadas no mostraron actividad antifúngica in vitro sobre los aislamientos de C. albicans y C. no albicans, por lo que fueron considerados resistentes. El promedio de las zonas de inhibición $(\mathrm{mm})$ que mostraron las cepas de Candida albicans, no albicans y cepas ATCC a las diferentes concentraciones de $\mathrm{NaOCl}$ se muestran en las Tablas I, II, III respectivamente. El efecto antifúngico del $\mathrm{NaOCl}$ sobre los aislamientos de $C$. albicans fue dosis dependiente, mostrando un halo de inhibición mayor a una concentración de 0,5\% (27 mm) el cual disminuyó conforme disminuyó la concentración, obteniendo un halo de inhibición de $13 \mathrm{~mm}$ a una concentración de $0,062 \%$ de $\mathrm{NaOCl}$.

Con respecto a las cepas ATCC se obtuvo un halo de inhibición ligeramente mayor con respecto a C. albicans obtenida de prótesis dentales ( $31,5 \mathrm{~mm}$ vs $27 \mathrm{~mm}$ ) a una concentración de $0,5 \%$ de $\mathrm{NaOCl}$. Sin embargo a concentraciones de $0,125 \%$ y $0,062 \%$ las 
CASTILLO, S. D. M.; TELLO, Z. M. C.; SÁNCHEZ, V. L. O.; GÓMEZ, G. M. B.; NAVA-ZÁRATE, N. \& ARANDA-ROMO, S. Susceptibilidad in vitro de Candida albicans y no albicans aisladas de prótesis dentales de pacientes con estomatitis protésica a tres sustancias de desinfección. Int. J. Odontostomat., 9(3):373-377, 2015.

Tabla I. Medidas de los halos de inhibición obtenidos de los aislamientos de C. albicans provenientes de prótesis dentales a las diferentes concentraciones de $\mathrm{NaOCl}$.

\begin{tabular}{lcccc}
\hline \multirow{2}{*}{ Especies de Candida } & \multicolumn{4}{c}{ Solución desinfectante NaOCI } \\
\cline { 2 - 5 } & $\mathbf{0 , 5 \%}$ & $\mathbf{0 , 2 5} \%$ & $\mathbf{0 , 1 2 5 \%}$ & $\mathbf{0 , 0 6 2} \%$ \\
\hline C. albicans & $32^{*}$ & 19 & 17 & 15 \\
C. albicans & 24 & 18 & 13 & 11 \\
C. albicans & 25 & 16 & 10 & 13 \\
Promedio & 27 & 17,6 & 13,3 & 13 \\
\hline
\end{tabular}

*Los números representan los diámetros de las zonas de inhibición en milímetros.

Tabla II. Medidas de los halos de inhibición obtenidos de los aislamientos de cepas ATCC provenientes de prótesis dentales a las diferentes concentraciones de $\mathrm{NaOCl}$.

\begin{tabular}{lcccc}
\hline \multirow{2}{*}{ Especies de Candida } & \multicolumn{4}{c}{ Solución desinfectante NaOCI } \\
\cline { 2 - 5 } & $\mathbf{0 , 5 \%}$ & $\mathbf{0 , 2 5 \%}$ & $\mathbf{0 , 1 2 5 \%}$ & $\mathbf{0 , 0 6 2} \%$ \\
\hline ATCC24433 & $28^{*}$ & 27 & 13 & 13 \\
ATCC90028 & 35 & 13 & 11 & 7 \\
Promedio & 31,5 & 20 & 12 & 10 \\
\hline
\end{tabular}

*Los números representan los diámetros de las zonas de inhibición en milímetros.

Tabla III. Medidas de los halos de inhibición obtenidos de C. no albicans provenientes de prótesis dentales a las diferentes concentraciones de $\mathrm{NaOCl}$

\begin{tabular}{lcccc}
\hline \multirow{2}{*}{ Especies de Candida } & \multicolumn{4}{c}{ Solución desinfectante $\mathbf{N a O C l}$} \\
\cline { 2 - 5 } & $\mathbf{0 , 5 \%}$ & $\mathbf{0 , 2 5 \%}$ & $\mathbf{0 , 1 2 5 \%}$ & $\mathbf{0 , 0 6 2} \%$ \\
\hline C. no albicans & $19^{*}$ & 13 & 11 & 9 \\
C. no albicans & 20 & 14 & 8 & 6 \\
C. no albicans & 13 & 11 & 9 & 7 \\
Promedio & 17,3 & 12,6 & 9,3 & 7,3 \\
\hline
\end{tabular}

*Los números representan los diámetros de las zonas de inhibición en milímetros.

Tabla IV. Comparación de los halos de inhibición entre C. albicans, C. no albicans y Cepas ATCC.

\begin{tabular}{lccc}
\hline Solución & \multicolumn{3}{c}{ Especies de Candida } \\
\cline { 2 - 4 } desinfectante & Cepas ATCC & C. albicans & C. no albicans \\
\hline NaOCI 0,5\% & $31,5^{*}$ & 27 & 17,3 \\
NaOCI $\mathbf{0 , 2 5 \%}$ & 20 & 17,6 & 12,6 \\
NaOCl 0,125\% & 12 & 13,3 & 9,3 \\
NaOCI 0,062\% & 10 & 13 & 7,3
\end{tabular}

*Los números representan el promedio en milímetros obtenido de cada cepa evaluada.

cepas ATCC mostraron un menor halo de inhibición con respecto a $C$. albicans (12 mm vs $13,3 \mathrm{~mm}$ y $10 \mathrm{~mm}$ vs $13 \mathrm{~mm}$ ).

Los halos de inhibición observados sobre las cepas no albicans de igual manera fueron dosis dependiente, mostrando un halo de 17,3 $\mathrm{mm}$ a una concentración de $0,5 \%$ el cual disminuyó a 7,3 $\mathrm{mm}$ a una concentración de $0,062 \%$. A todas las concentraciones los halos de inhibición mostraron un menor diámetro con respecto a $C$. albicans y cepas ATCC.
La comparación de los promedios obtenidos de los halos de inhibición de las especies evaluadas se muestra en la Tabla IV. Podemos observar que el efecto antifúngico del $\mathrm{NaOCl}$, fue mayor en las cepas ATCC a las diferentes concentraciones excepto a una concentración de $0,062 \%$, a esta concentración el mayor efecto antifúngico se obtuvo sobre las especies de C. albicans. El efecto antifúngico fue medido en base al tamaño de los halos de inhibición de tal forma que se consideró al mayor halo de inhibición que se obtuvo por cada dosis como el efecto antifúngico más eficaz. Con respecto a la susceptibilidad que mostraron las cepas no albicans al $\mathrm{NaOCl}$ se determinó que fueron las menos sensibles debido a que fueron las que obtuvieron los menores halos de inhibición a las diferentes concentraciones.

\section{DISCUSIÓN}

En el presente trabajo reportamos la susceptibilidad antifúngica in vitro, que muestran aislamientos clínicos de Candida obtenidos de prótesis dentales a 3 sustancias utilizadas como desinfectantes. En primer lugar determinamos la susceptibilidad antifúngica in vitro al $\mathrm{AC}$ el cual no presentó ningún halo de inhibición, esto tal vez debido a que las concentraciones utilizadas no fueron las adecuadas para observar el efecto, este mismo fenómeno lo observaron Pinto et al., los cuales reportaron que una solución de $A C$ al $10 \%$ no eliminó $C$. albicans de las prótesis. Sin embargo otros estudios in vitro han demostrado que dosis bajas del ácido acético son fungicidas, ya que inducen la apoptosis en $C$. albicans, la inmersión total de dentaduras en una solución de AC al $10 \%$ o incluso $5 \%$ durante la noche, reduce las unidades formadoras de colonia (UFC/ml) de Candida spp. en saliva, así como la 
CASTILLO, S. D. M.; TELLO, Z. M. C.; SÁNCHEZ, V. L. O.; GÓMEZ, G. M. B.; NAVA-ZÁRATE, N. \& ARANDA-ROMO, S. Susceptibilidad in vitro de Candida albicans y no albicans aisladas de prótesis dentales de pacientes con estomatitis protésica a tres sustancias de desinfección. Int. J. Odontostomat., 9(3):373-377, 2015.

presencia de EP. El efecto de remoción de la solución de $\mathrm{AC}$ al $5 \%$ o $10 \%$ es comparable con una solución de $\mathrm{NaOCl}$ al $1 \%$ (Ali-Jafari et al., 2012).

Por otra parte la SSO con pH neutro en nuestro estudio no mostró inhibición alguna en las cepas de C. albicans y no albicans, sin embargo publicaciones previas han demostrado su efecto microbicida (Zeng et al.; lieri et al.) Por ser producto de aparición reciente, cuenta con escasos reportes en la literatura, son necesarios más estudios para demostrar su eficacia.

Finalmente el desinfectante reconocido como "Estándar de oro" para la desinfección de prótesis colonizadas por Candida, es el $\mathrm{NaOCl}$, en este estudio pudimos corroborar su eficacia, ya que mostró la mejor susceptibilidad antifúngica in vitro, esto debido a que fueron utilizadas las concentraciones adecuadas, previamente reportadas en la literatura, siendo la concentración al 0,5\% la que mostró la mayor eficacia, esto coincide con lo reportado previamente (Skupien et al., 2013) quienes analizaron diversos estudios in vitro, en donde se demostró que está concentración es eficaz para desinfectar la superficies de las prótesis, por otro lado esta misma concentración fue evaluada in vivo por de Sousa Porta et al. (2013), quienes demostraron su eficacia en la reducción de microorganismos sin causar cambios significativos en el color o la rugosidad de la prótesis. En este sentido, para demostrar el efecto que tiene el $\mathrm{NaOCl}$ sobre la resina acrílica de la prótesis, McNeme et al. (1991) mostraron que no existen cambios de color en las resinas acrílicas después del uso de $\mathrm{NaOCl}$ en distintas concentraciones $(0,525 \%, 1 \%$ y $5,25 \%)$, en un lapso de $72 \mathrm{~h}$. Orsi \& Andrade (2004) reportaron que no hay afecciones en la fuerza de flexión de la prótesis después de una inmersión en $\mathrm{NaOCl}$ al $1 \%, 2,5 \%$ y $5,25 \%$, en un lapso de inmersión de $10 \mathrm{~min}$. Sin embargo debido al sabor y olor desagradable del $\mathrm{NaOCl}$ a estas concentraciones tan elevadas, la prótesis debe enjuagarse adecuadamente para remover el producto, además de que existe el riesgo de quemaduras en la mucosa de soporte de la prótesis, por tal motivo es preferible utilizar concentraciones más bajas demostrando su eficacia in vitro como lo reportado previamente por Davi et al., donde describe que concentraciones al $1 \%$ de $\mathrm{NaOCl}$ son desinfectantes sin embargo son suficientes para influir en la estabilidad, color y fuerza de flexión de la prótesis, por lo tanto nosotros demostramos in vitro que concentraciones menores al $1 \%$ tienen buena actividad antifúngica.
Las cepas ATCC mostraron la mayor eficacia antifúngica, esto debido a que son cepas de referencia las cuales sirven de controles, no obstante los aislamientos recuperados de prótesis dentales debido a las condiciones y edad de las prótesis activan factores de virulencia y capacidades que les permiten su supervivencia a cualquier agente desinfectante, más aún cuando se encuentran en el biofilm (Sánchez et al., 2013). Por otro lado identificamos que las especies sobre las cuales el $\mathrm{NaOCl}$ mostró una menor eficacia fueron las $C$. no albicans, esto es de gran relevancia ya que las especies que se aíslan con mayor frecuencia de las prótesis según el estudio realizado por Nayak et al. (2012) son las especies de Candida no albicans.

Una de las limitaciones de nuestro estudio fue el número de cepas evaluadas y la metodología utilizada ya que al ser un método cualitativo, no permite determinar las concentraciones mínima inhibitorias de las sustancias desinfectantes, una de las expectativas del estudio es evaluar estas sustancias por el método de microdiluciones, con un mayor número de aislamientos y además llevar a cabo ensayos clínicos controlados aleatorizados para demostrar su eficacia in vivo. El NaOCl al 0,5\% mostró la mejor inhibición in vitro contra $C$. albicans y Candida no albicans, esta inhibición fue dosis dependiente.

CASTILLO, S. D. M.; TELLO, Z. M. C.; SÁNCHEZ, V. L. O.; GÓMEZ, G. M. B.; NAVA-ZÁRATE, N. \& ARANDA-ROMO, S. In vitro susceptibility of Candida albicans and non-albicans isolated from dental prothesis in patients with denture stomatitis to three disinfectants. Int. J. Odontostomat., 9(3):373-377, 2015.

ABSTRACT: Despite the large number of products available for denture cleaning, less than $60 \%$ of denture wearers use them, possibly due to the economic situation. The aims were to determine the in vitro anti-fungal susceptibility of Candida species to three denture disinfectants (Sodium hypochlorite, acetic acid and super oxidized solution). Clinical isolates were obtained from denture wearers diagnosed with denture stomatitis for its posterior in vitro susceptibility study to the different substances. $0.5 \%$ Sodium hypochlorite showed the higher in vitro inhibitory effect on Candida albicans and non-albicans. Acetic acid and super oxidized solution showed no inhibition in both species. $0.5 \%$ Sodium hypochlorite has an in vitro inhibitory effect on Candida species.

KEY WORDS: Candida albicans, denture stomatitis, sodium hipoclorite. 
CASTILLO, S. D. M.; TELLO, Z. M. C.; SÁNCHEZ, V. L. O.; GóMEZ, G. M. B.; NAVA-ZÁRATE, N. \& ARANDA-ROMO, S. Susceptibilidad in vitro de Candida albicans y no albicans aisladas de prótesis dentales de pacientes con estomatitis protésica a tres sustancias de desinfección. Int. J. Odontostomat., 9(3):373-377, 2015.

\section{REFERENCIAS BIBLIOGRÁFICAS}

Ali-Jafari, A.; Falah-Tafti, A.; Hossein, M.; Zahraeii, A. \& Kazemi, A. Vinegar as a removing agent of Candida albicans from acrylic resin plates. Jundishapur $J$. Microbiol., 5(2):388-92, 2012.

Chau, V. B.; Saunders, T. R.; Pimsler, M. \& Elfring, D. R. Indepth disinfection of acrylic resins. J. Prosthet. Dent., 74(3):309-13, 1995.

Davi, L. R.; Peracini, A.; Ribeiro Nde. Q.; Soares, R. B.; da Silva, C. H.; Paranhos, Hde. F. \& de Souza, R. F. Effect of the physical properties of acrylic resin of overnight immersion in sodium hypochlorite solution. Gerodontology, 27(4):297-302, 2010.

de Sousa Porta, S. R.; de Lucena-Ferreira, S. C.; da Silva, W. J. \& Del Bel Cury, A. A. Evaluation of sodium hypochlorite as a denturecleanser: a clinical study. Gerodontology, 32(4):260-6, 2015.

Estrela, C.; Ribeiro, R. G.; Estrela, C. R.; Pécora, J. D. \& Sousa-Neto, M. D. Antimicrobial effect of $2 \%$ sodium hypochlorite and $2 \%$ chlorhexidine tested by different methods. Braz. Dent. J., 14(1):58-62, 2003.

Ileri, C.; Sezen, Y. \& Dimoglo, A. Investigation of the efficacy of electrolyzed acid water on the standard strains of some pathogenic microorganisms. Mikrobiyol. Bul., 40(4):31724, 2006.

Jackson, S.; Coulthwaite, L.; Loewy, Z.; Scallan, A. \& Verran, $\mathrm{J}$. Biofilm development by blastospores and hyphae of Candida albicans on abraded denture acrylic resin surfaces. J. Prosthet. Dent., 112(4):988-93, 2014.

Koch, C.; Bürgers, R. \& Hahnel, S. Candida albicans adherence and proliferation on the surface of denture base materials. Gerodontology, 30(4):309-13, 2013.

McNeme, S. J.; von Gonten, A. S. \& Woolsey, G. D. Effects of laboratory disinfecting agents on color stability of denture acrylic resins. J. Prosthet. Dent., 66(1):132-6, 1991.

Nayak, S.; Kavitha, B.; Sriram, G.; Saraswathi, T. R.; Sivapathasundharam, B. \& Dorothy, A. L. Comparative study of Candida by conventional and CHROMagar method in non-denture and denture wearers by oral rinse technique. Indian J. Dent. Res., 23(4):490-7, 2012.

Orsi, I. A. \& Andrade, V. G. Effect of chemical disinfectants on the transverse strength of heat-polymerized acrylic resins submitted to mechanical and chemical polishing. J. Prosthet. Dent., 92(4):382-8, 2004.
Sánchez-Vargas, L. O.; Estrada Barraza, D.; Pozos-Guillen, A. J. \& Rivas-Caceres, R. Biofilm formation by oral clinical isolates of Candida species. Arch. Oral Biol., 58(10):131826, 2013.

Skupien, J. A.; Valentini, F.; Boscato, N. \& Pereira-Cenci, T. Prevention and treatment of Candida colonization on denture liners: a systematic review. J. Prosthet. Dent., 110(5):356-62, 2013.

Pinto, T. M.; Neves, A. C.; Leão, M. V. \& Jorge, A. O. Vinegar as an antimicrobial agent for control of Candida spp. in complete denture wearers. J. Appl. Oral Sci. Rev., 16(6):385-90, 2008.

Williams, D. W. \& Lewis, M. A. Isolation and identification of Candida from the oral cavity. Oral Dis., 6(1):3-11, 2000.

Williams, D. W.; Kuriyama, T.; Silva, S.; Malic, S. \& Lewis, M. A. Candida biofilms and oral candidosis: treatment and prevention. Periodontol. 2000, 55(1):250-65, 2011.

Zeng, X.; Ye, G.; Tang, W.; Ouyang, T.; Tian, L.; Ni, Y. \& Li, P. Fungicidal efficiency of electrolyzed oxidizing water on Candida albicans and its biochemical mechanism. J. Biosci. Bioeng., 112(1):86-91, 2011.

Dirección para Correspondencia:

Saray Aranda-Romo

Laboratorio de Bioquímica, Microbiología y Patología

Facultad de Estomatología

Universidad Autónoma de San Luis Potosí

San Luis Potosí

MÉXICO

Email: aran_sa77@hotmail.com

Recibido : 07-03-2015

Aceptado: 03-10-2015 